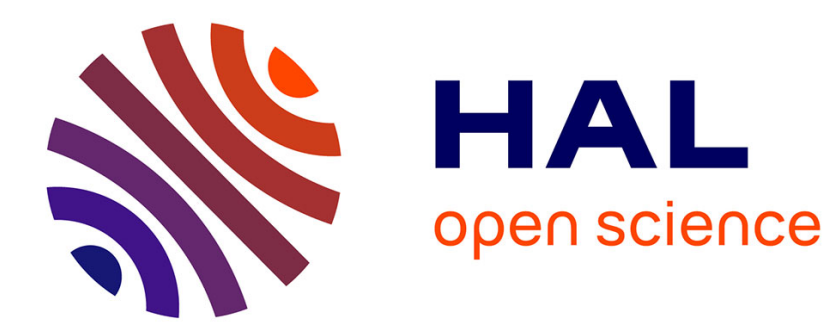

\title{
Mind-Body Connection and Causation: Conceptual and Experimental Advances
}

\author{
Pierre Uzan, Université Paris
}

\section{To cite this version:}

Pierre Uzan, Université Paris. Mind-Body Connection and Causation: Conceptual and Experimental Advances. Foundations of Science, 2020. hal-03189186

\section{HAL Id: hal-03189186 \\ https://hal.science/hal-03189186}

Submitted on 2 Apr 2021

HAL is a multi-disciplinary open access archive for the deposit and dissemination of scientific research documents, whether they are published or not. The documents may come from teaching and research institutions in France or abroad, or from public or private research centers.
L'archive ouverte pluridisciplinaire HAL, est destinée au dépôt et à la diffusion de documents scientifiques de niveau recherche, publiés ou non, émanant des établissements d'enseignement et de recherche français ou étrangers, des laboratoires publics ou privés. 


\title{
Mind-Body Connection and Causation: Conceptual and Experimental Advances
}

\author{
Pierre Uzan \\ Chercheur associé au laboratoire CHArt, Cognition Humaine et Artificielle \\ Université Paris 8
}

\begin{abstract}
This article deals with the difficulties of the yet intuitive causal interpretation of the mindbody connection emphasized by metaphysical, theoretical and experimental considerations. It shows that a decisive contribution to determining the nature of this connection can be provided experimentally. This experimental test is designed within the framework of a general systems theory capable of representing the concepts of complementarity and entanglement that are involved in the description of the mind-body connection.
\end{abstract}

Key-words: Mind-Body Problem; Causation; Generalized Quantum Theory; Entanglement

\section{Introduction: the mind-body connection}

Each of us experiences daily the very strong correlation between our subjective experience and the change in our body, for example between the experience of fear and the acceleration of the heart rate. Brain imagery pinpoints the neurophysiological correlates of perception, emotions and even states of consciousness (Koch 2006) (Leopold and Logothetis 1996). Psychosomatic medicine establishes links between the physical diseases that we develop and our psychic life (Alexander 1950) (Groddeck 1923), extending Hippocrates' line of thought according to which disease is a loss of harmony between the body and the mind that could be restored by taking into account the "temperament" and the patient's experience. However, if we know for a long time that our subjective experience is strongly correlated with the physiological, nervous and endocrine activity of our body, to the point of rightly speaking of "psychosomatic unity" of the individual (Heinroth 1818) (Trombini and Baldoni 2005), the question about the nature of these correlations is still an object of controversy. How could we make intelligible the mind-body connection? 
Very different responses to this long-standing question have been proposed. The response supported by most contemporary biologists is to say that our thoughts and emotions are nothing but neural processes (Crick 1994) (Minsky 1988) or the informational processes they give rise to (Tononi 2007). It is the case of the "reentry" model proposed by Edelman (2000), the informational model of consciousness proposed by Tononi (2007) or that of the global workspace proposed by Dehaene and his collaborators (Dehaene \& Changeux 2004). In other words, we could simply reduce or even "identify" our mental life to neurophysiological or informational processes. However, as Chalmers (2007) or Levine (1983) have noted, this reductionist materialist position inherited from Hobbes and recently supported by Kim (2006) cannot explain the subjective experience that accompanies these brain processes. This is what Chalmers calls the "hard problem of consciousness" (Chalmers 2007): how can brain processes "give rise" to subjective experience? A third-person description of how our brain works when we perceive a red rose cannot account for "what it is like" to perceive this red rose, which is our subjective experience associated with our brain functioning (Nagel 1974). Consequently, unless relegating subjective experience to the rank of "illusion", as proposed by Dennett (1991), this reductionist position seems to rest on an act of faith: the ultimate explanation of subjective experience will certainly be in terms of neurophysiological processes since we are (only) "neuronal human beings" (Changeux 2003).

In contrast, dualism upholds the irreducibility of the mental to the physical and has been developed according to two different ways: dualism of substances (Descartes 1641) or dualism of properties (Davidson 1970) (Jackson 1982), this latter position being generally explored within a materialist framework, hence conceiving mental properties as "emerging" from the brain (Searle 1997) (Sperry 1983) or "supervening" on their physical basis (Davidson 1970) (Kim 2006). However, as will be explained in this article, the yet intuitive causal interpretation of these correlations by dualism also leads to great difficulties. This causal interpretation has been questioned in the traditional metaphysical debates and by the logico-philosophical analysis of contemporary philosophers. On more experimental grounds, it has been questioned by Cannon-Bard's theory of emotions and, more recently, by Libet's experiments and its contemporary extensions. This paper aims to show that an important, even decisive, contribution to the determination of the mind-body connection can be provided by an experimental test designed within a general systems theory allowing the representation of the concepts of (generalized) complementarity and entanglement inherent to the description of the psychosomatic unity of the individual. This new conceptual framework is a generalized 
version of quantum theory that does not exclusively deal with the material realm but applies to any kind of systems.

Section II will begin by reminding the reader of the difficulties of the causal interpretation of the mind-body connection from a logico-philosophical analysis and from Cannon-Bard's theory of emotions. Section III will present some recent experimental investigations of the causal interpretation of the mind-body connection: Section III.1 will mention Libet's experiments and their recent extensions by Wegner (1999) and by Soon (2008); section III.2 will present an experimental test of the nature of the mind-body connection designed within this new general systems theory.

\section{The difficulties of the causal interpretation of the mind-body connection}

We intuitively think that the mind-body correlations are (possibly complex) "causal" relationships by referring to the possibility of voluntary movements: our will to raise an arm seems to really "cause" the rising of this arm. Reciprocally, it seems that we can assert without any doubt that a strike on our body "causes" a subjective sensation of pain. Even more, the aforementioned intertwinement between emotional and physical states, when it is recognized, is generally interpreted in terms of a complex or "multifactorial" causal process. However, if the concept of causation plays a central role in everyday life and is a touchstone for explaining phenomena in science, the question of building a concept of causation susceptible to be applied to the mind-body connection has given rise to many discussions.

\section{1. The questioning of mental causation by logico-philosophical arguments}

Let us first note that the mainstream physicalist position mentioned in Introduction avoids the difficulties of the causal interpretation of the mind-body connection because it merely reduces the action of the mental on the physical to material or physical causation, which is ruled by the well-known laws provided by natural science. However, if the concept of mental causation does not raise any difficulty here, this position leads to the not less essential problem of explaining the subjective experience that accompanies these neural processes - as explained in Introduction. This reductionist position that gets rid of subjective experience will then not be considered further in this article. We will concentrate on the difficulties of interpreting causally the mind-body connection for the dualist positions, which, at least phenomenally, recognize the heterogeneity of mental and bodily properties, events or processes. 
Descartes' dualism of substances (Descartes 1641), according to which the world would be composed of two distinct substances, the thinking substance (mind) and the extended substance (matter), as well as its contemporary formulations in terms of process (Beck \& Eccles 1992), leads to a solution of the mind-body problem calling for an elusive interaction between these two substances or these two types of heterogeneous processes. This interaction, which takes place in the pineal gland for Descartes and at the synaptic level for Beck and Eccles, is "elusive" because it refers to hypothetical mind-matter interaction laws. What could be the mediator of such "interaction"? If, for Descartes, this interaction can only be experienced but not conceptualized (Descartes 1644), some models of such an interaction have been recently proposed within the framework of quantum physics (Walker 1975) (Mattuck 1980). In Walker's model, it is the information processed by consciousness that would trigger physical (quantum) transitions, the amount of information involved in this psychokinetic "effect" being evaluated in terms of the Shannon's statistical information associated with its probability of occurrence. However, the subjectivist interpretation of the collapse of the wave function on which these models crucially rely can be easily challenged for its unclarity, its lack of coherence and by the fact that other justifications of this process have been proposed without appealing to the existence of a mental substance (Uzan 2013, chap. VII).

Non-reductionist physicalism (Davidson 1970) (Searle 1997) (Woodward 2003) tries to preserve the autonomy and the efficiency of the mind in a physicalist framework. However, if it does not speak explicitly of "interaction" between mind and body, it cannot provide a coherent definition of the concept of mental causation, as Kim showed with his argument of exclusion (Kim 2006). This author has explained that the causal closure of the physical world, which grounds physicalism, excludes the causal efficacy of the mental on the physical since the physical cause of a physical event necessarily preempts its alleged mental cause. Attempts to save mental causation within non-reductionist physicalism appeal to other interpretations of causation, such as the interventionist interpretation (Woodward 2003) and the counterfactual interpretation (Shapiro \& Sober 2007). The latter interpretations empty the notion of mental causation of any specificity that could distinguish it from that of correlation - by defining it, for example, as Kim suggests (Kim 2006), in terms of "generation or production" and not as mere correlations of changes or occurrences. Furthermore, as shown by Baumgartner (2010), the interventionist interpretation of causation is inapplicable to this domain insofar as, by the 
very definition of what is the "physical basis" of a mental event ${ }^{1}$, an intervention on the supposed mental cause cannot leave its physical basis unchanged, contrary to what founds this interpretation of causation (Woodward 2003).

The causal efficacy of the mental, and even the very consistency of the concept of mental causation is thus seriously questioned by the logico-philosophical analysis. In addition, as will be shown in the next section, this consistency can be questioned from more specific considerations about emotions.

\section{II.2. The questioning of the mind-body causation by Canon-Bard's theory of emotions}

We will here focus on the theories of emotions that explicitly deal with the question of the mind-body connection -in contrast with Darwin's evolutionary theory (Darwin 1872). William James suggested that emotion is nothing but the awareness of the physical changes activated by a stimulus. James-Lange's theory of emotions, proposed independently by William James (1884) and Carl Lange (1895), asserts that the phenomenal aspects of emotions, like the experience of fear or anger, originate from physiological reactions to stimuli and thus follow these visceral disturbances. As we experience different life situations, our nervous system gives rise to physical reactions associated with them, like for example increased heart rate or tremors, which would be "interpreted" or consciously perceived as subjective feelings. James-Lange' theory conceives emotional experience as originating from physiological signals sent by all parts of the organism, particularly by the viscera, in reaction to life situations. Along this line of thought, let us mention Damasio's purely physiological definition of emotions as (Damasio 1999, p. 280):

... many changes in the chemical profile of the body, as well as changes in the viscera and degree of contraction of the various striated muscles of the face, throat, trunk and limbs. But they also depend on the transformations undergone by all the neuronal structures at the origin of such changes and are likely to modify in return several neural circuits of the brain itself.

In agreement with James-Lange's theory, Damasio supports the view according to which the emotions, generated in extra-cortical structures from the information they receive about the state of our body, would be causally responsible for the constitution of the feeling of self: this influx of emotions is sent to the cortex which integrates it into all of its cognitive or motor tasks and thus gives the feeling that we are the actors of these experiences.

\footnotetext{
${ }^{1}$ Such a physical basis of any mental event must be supposed by materialism to ensure that mental events are always dependent and even determined by physical events.
} 
However, physiologist Walter Canon (1927) has pointed out several objections to JamesLange's theory that seem to severely question the causal interpretation of the connection from the physiological changes in the body to the emotional experience. He has first emphasized the fact that there is no one-to-one correspondence between them because of the nonspecificity of the organic changes that can accompany different emotions: the same visceral changes can be associated with different emotional experiences and even with non-emotional states. For example, if fear is associated with higher heart rate and increased sweating, the same physiological reactions can accompany other emotions, such as anger, and even a priori non-emotional situations like that of fever. Consequently, visceral changes cannot be regarded as causally responsible for specific emotional experiences since the same physiological changes can be associated to many different emotional experiences. Moreover, Canon has noticed that emotional, subjective responses occur too quickly to simply be caused by visceral changes (Cannon 1920) since the latter emotional experience response to the viscera changes would take approximately 3 seconds, which is far too long in comparison to the observed delays of affective responses to different stimuli -which weakens a bit more this causal interpretation. More radically, Cannon (1927) has shown on experimental grounds that emotions can be experienced without any visceral feedback to the brain: the removal of the sympathetic nervous system of a cat, which ensures the connection between the viscera and the central nervous system, had no significant effect on its emotional behavior. In contrast with James-Lange view, Cannon-Bard's theory of emotions then asserts that the seat of emotion lies in subcortical regions ${ }^{2}$ from which parallel innervations start, triggering physiological (peripheral) activity and "giving rise to" the subjective emotional experience (Cannon 1920) (Bard 1930). Consequently, according to Canon's theory, the psychological experience of emotion and the associated physiological changes can occur simultaneously and one of them cannot then cause the other.

Can the theories of emotions that explicitly refer to the cognitive aspect of consciousness support the causal physiological-to-mental interpretation of emotions? Let us for example mention Schachter-Singer's theory (1962) according to which emotion would result from the "interaction" between a physiological arousal and the causal attribution of this arousal to some features of the stimuli, which is of cognitive order. In such theories of cognitive assessment, emotional experience relies on personal significance assigned to the situation lived by the subject and its past experiences (Lazarus 1991). These theories explicitly refer to

\footnotetext{
2 Originally, Cannon assigned this role to the thalamus and Bard to the hypothalamus, but now this role is assigned to the whole limbic system that groups all the subcortical structures involved in by the generation of emotions (MacLean 1949).
} 
a notion of signification associated to life situations, along Groddeck's and Broom's line of thought according to which some "meaning-full" diseases could be regarded as the somatic expression, the material signifier, of the patient's lived experience and emotional suffering (Groddeck 1923) (Broom 2007). However, besides this consistent representation of emotions that define emotional experience in terms of meaning, there is still no clear conclusion neither on the temporal order of their bodily and mental expressions nor on the supposed "causal" nature of their connection. Actually, if subjective and physiological aspects of emotions must be considered together in order to fully characterize them, the question of the nature of their connection, closely related to that of the existence of a temporal order in their appearance does not find a consensual response. As mentioned above, James-Lange's theory of emotion asserts that feelings are the result of physiological changes triggered by a stimulus. This view is supported by Damasio's biological approach according to which physiological changes would be causally responsible for the constitution of the feeling of self. On the other hand, in Cannon-Bard's theory of emotions the psychological experience of emotion and the associated physiological changes are not causally related and can occur simultaneously.

As a matter of fact, contradictory observations seem to confirm Cannon-Bard's conclusion since both temporal orders have been reported. In some cases, it seems clear that the physiological changes caused by taking a medication is followed by a subjective experience. For example, negative feelings associated with anxiety are diminished after neurological changes (in the release of neuromodulators) induced by the taking of an anxiolitic, and the physiological changes triggered by the stimulation of certain points of acupuncture have subsequent anti-depressive effects (Luo et al., 1990). While in other cases it has been observed that purely psychological factors can be followed by real physiological changes. It is for example the case in the placebo "effect" where the patient's trust in the physician and in the prescribed drug can have spectacular physiological effects, such as eradicating pain or curing a duodenal ulcer (Janssen 2006). It has also been observed that a psychological stress gives rise to a subsequent overdrive of the sympathetic nervous system, which triggers an increase of adrenalin and cortisol production that have measurable, generally harmful, effects on the body (Selye 1978). Even more, a simple memory or the anticipation of a future difficult situation, which generates negative emotions, can be followed by important physiological changes, like for example physical symptoms of anxiety -increasing heart rate and blood pressure, sweaty palms, dry mouth or shaky hands. Subsequently, as suggested by Cannon-Bard's theory and in agreement with the logico-philosophical analysis of section II.1, it seems that no causal order between the subjective expression of an emotion and the 
associated bodily changes can be definitely and universally asserted. Generally speaking, what can be said is that the thorough interdependence of the psychic and the somatic features of any phenomenon experienced by a person cannot be consistently interpreted as a direct causal connection. This conclusion will find an experimental confirmation (see section III.2). A new a-causal interpretation of the mind-body connection that explicitly accounts for the existence of global, psychophysical experiences then seems to be required.

\section{Recent experimental investigations on mind-body causation}

Beyond the long-standing metaphysical and theoretical debates on the nature of the mindbody connection, the experimental approach mentioned below has revealed very interesting features of this connection. Let us mention these experimental investigations and their sometimes surprising consequences.

\section{III.1. Is mental causation an illusion?}

Experimental investigations have shown that an essential distinction between the feeling that our conscious intentions can be fully responsible for our actions and their real causal responsibility must be done.

Libet's experiments (Libet 1983; 1985) have first shown that when a subject takes the conscious decision to move his or her body the regions of its brain susceptible to trigger this movement has already been activated. This activation of the brain, signaled by a "readiness potential", occurs about $350 \mathrm{~ms}$ before the conscious decision. These experiments seem to show that the conscious decision would only be an epiphenomenal, psychological manifestation of a previous unconscious brain activity that actually decides to perform the movement. Generally speaking, they seem to undermine the idea that our conscious intentions could really cause our acts, and then the very existence of free will: How can I freely decide something if my brain has already begun to act in the moment I am consciously choosing?

These results have been extensively discussed, regarding in particular the reliability of the moment the subject reports its conscious intention (Gomes 2002). One can for example mention William James' notion of "specious present" that assigns a kind of thickness to the present and could then mislead our perceptions of the moment of the conscious decision. However, a recent experiment performed by Soon and collaborators have confirmed and even refined these results (Soon et al. 2008). In this experiment, the subject can choose between pushing a button with his left hand or another one with his right hand, and decides on the moment to act. During the experiment, the subject's brain is observed by MRI, which allows 
to simultaneously analyze the activity of diverse brain areas. MRI imaging has shown us that preparatory brain activity exists 7 to 10 seconds before the subject makes a decision. Furthermore, this brain activity makes possible to predict, to a certain extent, on which side the subject will push the button. These results therefore seem to call into question even more radically the idea that our conscious intentions could really be "causally responsible" for our acts.

This line of thought has been explored by psychologist Daniel Wegner who has confirmed on experimental basis that our sense of free will does not reflect the underlying causes of our behaviors (Wegner 1999). In one of these experiments a subject is placed in front of mirrors positioned in a way that leads the subject to believe that what appears to be its arm is in fact someone else's. The subject then receives instructions to make certain arm movements and, immediately afterwards, the arm that the subject sees through the mirror scheme executes each of these movements. The subject then reports the impression of being at the origin of this illusory movement seen in the mirror. Wegner analyses these kinds of experiments as follows:

The experience of willing an act arises from interpreting one's thought as the cause of the act. Conscious will is thus experienced as a function of the priority, consistency, and exclusivity of the thought about the action. The thought must occur before the action, be consistent with the action and not be accompanied by other causes. ${ }^{3}$

Libet's experiments, its extensions by Soon et al. and Wegner's experiments thus seem to clearly establish a distinction between our feeling of causal efficacy of our conscious will and the supposed real causal connection between our thoughts and our actions. As pointed out by Wegner:

...it may be that people experience conscious will when they interpret their own thought as the cause of their action. This idea means that people can experience conscious will quite independent of any actual causal connection between their thoughts and actions. (Wegner 1999)

This essential distinction between the feeling that our conscious intentions can be fully responsible for our actions and their actual responsibility, which may even be completely independent, shows that the generally uncontested idea of a causal efficacy of the mind over the body and the world might only be a simple illusion. This conclusion provides us with a

\footnotetext{
${ }^{3}$ Wegner has shown on experimental basis that the feeling of causal responsibility of our conscious decision is generated by the satisfaction of three conditions: its temporal anteriority on the action (but not a too long one), its consistency with it and the apparent exclusion of any other potential cause of it. (Wegner 1999).
} 
necessary step back on our belief in the causal efficacy of the mental and thus encourages us to go further in the knowledge of the nature of the mind-body connection.

\section{III.2. An experimental test to determine the nature of the mind-body connection}

\section{Principle of the test}

A clear experimental response to the question of the nature of the mind-body connection can be provided by measuring the correlation degree between couples of complementary emotional observables and complementary bodily observables and by comparing this degree with two theoretical bounds derived from a very general systems theory obtained by generalizing quantum theory (see below the theoretical modeling). Two observables are said to be "complementary" in quantum theory (and in generalized quantum theory) if they are at least artially incompatible, which means that they cannot be measured simultaneously with accuracy, or, equivalently, that the order in which they are measured is not indifferent. For each of the two pairs of complementary emotional (A, B) and physiological (A ', B') observables chosen, the four combinations (A, A'), (A, B'), (A', B) and (A', B') are conjointly measured and recorded in order to calculate the so-called CHSH (for Clauser, Horne, Shimony and Holt) correlation factor R defined below, and to compare its absolute value with these theoretical bounds. The range of values of $|R|$ will inform us directly about the nature of the considered correlations ${ }^{4}$.

\section{Theoretical modeling}

The generalized version of quantum theory used to derive the relevant bounds keeps the algebraic structure of quantum theory allowing to represent the essential concepts of complementarity and entanglement that are necessary to deal with situations involving mental processes, as shown by Aerts and his team $(2011 ; 2020)$ and by Busemeyer and Bruza (2012). This algebraic structure is thus appropriate for dealing with the mind-body problem (Uzan 2014a). In this generalized version of quantum theory, the notion of "system" denotes any part

\footnotetext{
${ }^{4}$ This experimental test must not be understood as assessing "one-to-one" relationships between specific emotional variables and specific bodily variables, because it is a statistical one that involves many couples of mental-physiological observables and many subjects. Consequently, it can provide a very significant general information on the nature of the mind-body connection, and, in particular, on the validity of its causal interpretation.
} 
of reality susceptible of investigation and that of "observable" any property capable of being measured or observed in a reproducible manner, any a priori reference in the physical world being thus eliminated (Atmanspacher et al. 2002) (Walach and von Stilfried 2011). Like in standard quantum theory, the notion of complementary observables is represented by the noncommutativity of the corresponding operators and that of entanglement by the nonfactorability of the vector-state. It has been shown in (Uzan 2014; 2020) that Bell's test, its extensions to non-local correlations and its consequences regarding causation, which have already been successfully used in the strict quantum domain (Clauser and al 1969) (d'Espagnat1994) (Bhurman and Massar 2005), can be used to evaluate the nature of the correlations between the observables respectively defined on each of the two parts of such a generalized system. This means that the nature of these correlations can be determined by comparing the absolute value of the CHSH correlation coefficient (Clauser et al. 1969), defined by

$$
R=S\left(a a^{\prime}\right)+S\left(a b^{\prime}\right)+S\left(b a^{\prime}\right)-S\left(b b^{\prime}\right),
$$

where $S(a$ a') is the mean value of the product of outcomes of the joint measure of observables A and $\mathrm{A}^{\prime}$ (which have been normalized), and similarly for the other terms, with the following two theoretical bounds: the Bell bound, which is 2, marking the border between classical correlations due to a local determination and non-local correlations (Bell 1964), and an interaction bound, $\beta=\left(4+\|[\mathrm{A}, \mathrm{A} \text { '] [B', B] } \|)^{1 / 2}\right.$, which is calculated from the commutators $\left[\mathrm{A}, \mathrm{A}^{\prime}\right]=\mathrm{AA}^{\prime}-\mathrm{A}^{\prime} \mathrm{A}$ and $\left[\mathrm{B}, \mathrm{B}^{\prime}\right]=\mathrm{BB}^{\prime}-\mathrm{B}^{\prime} \mathrm{B}$ of the couples $\left(\mathrm{A}, \mathrm{A}^{\prime}\right)$ and $\left(\mathrm{B}, \mathrm{B}^{\prime}\right)$ of observables considered, marking the border between non-local correlations and causal interaction (Uzan2014a). If the absolute value $|R|$ of the correlation factor is greater than $\beta$ these correlations can be regarded as causal interactions and if $|R|$ is less than $\beta$, the correlations studied are no-signaling, they cannot be explained by the exchange of any signal between the two considered sub-systems. Moreover, if the absolute value $|\mathrm{R}|$ of the correlation factor is less than 2, the psychophysical correlations are classical local correlations, meaning that they are predetermined by local properties intrinsically possessed by the sub-systems considered (in particular, they can be regarded as common-cause correlations). The case $2<|R| \leq \beta$ means that they are no-signaling non-local correlations, which can be neither reduced to causal interaction nor to classical local (or common-cause) correlations. In this case, the individual psychosomatic state cannot be factored into a product of a single somatic state and 
a single mental state. It is this property of entanglement ${ }^{5}$ of psychosomatic states which would give rise to non-classical correlations between the psychic and somatic characteristics of the individual. All these theoretical developments are presented in more details in the references (Uzan 2014a; 2016; 2017; 2020).

The individual is here conceived as a unique and indivisible system, and its psychosomatic states are represented by unit vectors of the space of psychosomatic states, which is a vector space on the body of complex numbers provided with a scalar product (a complex Hilbert space). The space of psychosomatic states is generated by the tensor product of the "privileged" bases constituted by the eigenvectors of mental and somatic observables selected for each of these two domains. These observables form, for each of these domains, a noncommutative operator algebra acting on their respective space. The psychosomatic state of an individual can thus be represented formally by a vector of a Hilbert space written as a linear combination of somatic and mental "product states" considered as elementary.

\section{Previous meta-analysis}

In a previous meta-analysis published in Axiomathes (Uzan 2017) and partially presented in the Appendix, the physiological variables were measured continuously on subjects conditioned in specific emotional states, using conventional methods (Lang 1979), telling them stories or showing them videos related to their personal experience -their values being evaluated in relation to those corresponding to a "neutral" emotional conditioning. It was thus supposed that the conditioning was reliable enough to provide significant results, this reliability being checked by asking subjects to report on a scale the intensity of their induced emotions by performing an analysis of variances. This meta-analysis has already provided significant results since on 168 trials, 142 values of the CHSH correlation factor R satisfy the signaling bound (that is, $|\mathrm{R}| \leq \beta$ ). Assuming that the distribution of the values of $\mathrm{R}$ roughly respects a normal distribution, we can calculate that, with a confidence level of $95 \%, \mathrm{R}$ satisfies the signaling bound with a probability comprised between $78,9 \%$ and $90,1 \%$. This result then confirms the quasi-impossibility to interpret causally the studied psychophysical correlations with a high confidence level. However, these data were based on all-or-nothing emotional conditioning (subjects were "happy" or not, "angry" or not, ...), which means that the intensity of emotions was not measured. The consequence of the latter constraint is that all the situations where

\footnotetext{
${ }^{5}$ The concept of generalized entanglement, which is here assigned to the mind-body connection, generalizes that of quantum entanglement introduced by Schrödinger (1935) to characterize the nature of the correlations between two particles having interacted in their common past.
} 
emotional observables do not have a maximal intensity have been ruled out of this analysis, which surely skews the results. To allow for a more complete and more reliable test, one needs to consider joint measurements of continuous emotional variables, which evaluate the change in intensity of the emotions experienced by the subjects that accompanies the continuous changes in their physiological variables. Therefore, in order to improve the accuracy of the results emotional variables will also be measured continuously using a continuous response digital interface (CRDI) on which the subject moves a stylus or finger (Geringer et al. 2004).

\section{Practical realization.}

\section{Choice of pairs of complementary emotional and physiological observables:}

Complementary physiological observables can be defined from couples of combined physical quantities. For example, the duration of a signal and its spectral width, which are combined physical quantities, make it possible to define the pair of complementary observables (T, F) where $\mathrm{T}$ is an operator measuring the duration of a heartbeat provided by electrocardiogram and $\mathrm{F}$ is an operator measuring the spectral dispersion. Blood pressure and stroke volume, which is related to heart rate, can also be used, or the metabolic activity of the brain and pupillary diameter. For the emotional realm, we have a wide choice because the emotions are all complementary two by two to varying degrees. However, by choosing emotions with a high degree of incompatibility (such as anger and disgust), the results will be more significant (Uzan 2016).

Population: This statistical test will be carried out with a population of at least 200 volunteers, a population if possible sufficiently homogeneous, whose psychological and physiological characteristics, social and cultural origins are not too far apart, for the purpose of greater significance of the results.

Measuring equipment and instruments used: In addition to computers, devices for measuring physiological variables: ECG (and electrodes), devices for measuring electrodermal activity, sphygmomanometer, IR camera for measuring brain metabolic activity from the surface temperature changes. As mentioned above, emotional intensities will be measured by using a continuous response digital interface (CRDI) on which the subject moves a stylus or finger (Geringer et al. 2004). We need statistical software for the processing of 
experimental data as well as image or sound software for the induction of specific emotions (using a computer).

This experiment will be soon carried out in a Paris laboratory of psychophysiology by a team newly constituted. The realization of this experimental test by other, independent teams would provide still more significant results on the nature of the mind-body connection. An appeal to other teams interested in this project is thus launched.

\section{Conclusion and prospect.}

In this article, we have mentioned the difficulties faced by the causal, yet intuitive, interpretation of the mind-body connection from different complementary approaches as well as from some recent experimental advances on this question. In particular, we have proposed a statistical test aimed to determine directly the nature of the mind-body connection by measuring conjointly emotional and physiological variables and by comparing their correlation factor to two bounds derived from a general systems theory obtained by generalizing quantum theory. Preliminary results of this test, whose protocol will be ameliorated by measuring continuously emotional variables, have shown, with a great confidence degree, that the mind-body connection can hardly be interpreted as a mere causal interaction, whatever its form or its complexity. Generally speaking, it seems that all the presented considerations on the nature of the mind-body connection, being drawn from a logico-philosophical analysis, from theoretical reflections on emotions or from experimentation, lead us to conceive of the mind-body connection as a kind of parallelism of aspects, along Spinoza's neutral monism philosophy (Spinoza 1675). A new concept is then required in order to make intelligible the strong a-causal mind-body correlations, this concept being radically different from that of causation as well as from those of emergence and supervenience that have been proposed to save the irreducibility of the mental to the physical within a materialist framework. As has already been suggested in several publications (Uzan 2012; 2014a,b) (Walach \& Römer 2011) (Atmanspacher 2003), a good candidate is the concept of generalized entanglement built by generalizing that of quantum entanglement because it can explain the existence of strong mind-body correlations without facing the difficulties of the causal interpretation. The experiment proposed in section III.2 which has already provided preliminary results will test more accurately this hypothesis. 
In an article by Kassam and Mendes (2013), the section entitled "Cardiovascular Reactivity" (p. 6-7) reports the mean change scores $\left\langle\Delta Y_{i}\right\rangle$ of six cardiovascular variables $Y_{i}$ that are associated with two emotional conditioning (Fear and Anger) resulting from the realization of mental tasks, in the case the subjects report their emotion and in the case they do not report their emotion. The cardiovascular observables measure the $\Delta Y_{i}$ while the corresponding emotional observables measure the degree of anger and shame experienced by a subject. Due to the reliability of the method of conditioning (p. 5, section "Behavioral Coding", second paragraph), the measurement of a cardiovascular observable in a specified emotion can be considered as a joint measurement whose value of the emotion observable is 1 (state of "pure" emotion). These authors study the physiological effects of measuring emotion, showing that the act of reporting an emotion has an impact on the body's reaction.

However, like in the reference (Uzan 2017), we will here only focus on the case where the subjects do not report their emotion and use the corresponding data to compute the CHSH correlation factor for the different couples of cardiovascular-emotional observables. This article reports the change in HR (heart rate -in bpm), CO (cardiac output -in L/mn) and PEP (pre-ejection period -in ms), while the emotional observables under consideration are denoted by A (Anger) and Sh (Shame).

To compute $\mathrm{R}$ for all the couples of observables, we have first to compute the cardiovascular values normalized to unity. Table 1 provides the values and standard errors (SE) of the cardiovascular reactivity for the two possible emotional conditioning Anger and Shame ${ }^{6}$. The extremal values of cardiovascular variables that are needed for the normalization of these variables are also mentioned in the right column of this table:

\begin{tabular}{|l|l|l|l|}
\hline & $\mathrm{A}$ & $\mathrm{Sh}$ & Extremal values \\
\hline$<\Delta \mathrm{HR}>$ & $15.51, \mathrm{SE}=1.34$ & $10.13, \mathrm{SE}=1.4$ & 15,51 (Anger) \\
\hline$<\Delta \mathrm{PEP}>$ & $-11.29, \mathrm{SE}=1.20$ & $-5.51, \mathrm{SE}=1.26$ & $-11,29$ (Anger) \\
\hline$<\Delta \mathrm{CO}>$ & $0.96, \mathrm{SE}=.17$ & $0.36, \mathrm{SE}=.18$ & 0.96 (Anger) \\
\hline
\end{tabular}

Table 1. Mean changes and extremal values of cardiovascular variables for Anger and Shame

From table 1, we can compute the normalized value $\hat{Y}$ corresponding to each emotional conditioning (A or Sh) by using the following formula: $\hat{\mathrm{Y}}=\mathrm{Y}-\mathrm{Y}_{0} /\left|\mathrm{Y}_{\mathrm{extr}}-\mathrm{Y}_{0}\right|$, where

\footnotetext{
${ }^{6}$ These values are given in the section "Results", sub-section "Cardiovascular Reactivity" of this article of Kassam and Mendes.
} 
$\mathrm{Y}_{\text {extr }}$ and $\mathrm{Y}_{0}$ are, respectively, the extremal value of the variable $\mathrm{Y}$ and a reference value of $\mathrm{Y}$ (baseline value or a value corresponding to a "neutral" conditioning).

\begin{tabular}{|c|c|c|}
\hline & A & Sh \\
\hline$<\Delta \hat{\mathrm{H} R}>$ & 1 & $10.13 / 15.51=0.653$ \\
\hline$<\Delta \mathrm{PE \hat {P }}>$ & -1 & $-5.51 / 11.29=-0.488$ \\
\hline$<\Delta$ CÔ $>$ & 1 & $0.36 / 0.96=0.375$ \\
\hline
\end{tabular}

Table 2. Normalized values of cardiovascular variables

For example, the normalized value of $\langle\Delta \mathrm{HR}\rangle_{\mathrm{A}}$, which is the maximal value of the two reported values of $\mathrm{HR}$ (in A- and Sh-condition) is $\langle\Delta \hat{\mathrm{H} R}\rangle_{\mathrm{A}}=1$, while the normalized value of $\langle\Delta \mathrm{HR}\rangle_{\mathrm{Sh}}$ is $\langle\Delta \hat{\mathrm{HR}}\rangle_{\mathrm{Sh}}=\langle\Delta \mathrm{HR}\rangle_{\mathrm{Sh}} /\left|\langle\Delta \mathrm{HR}\rangle_{\mathrm{A}}\right|=10.13 / 15.51=0.653$. For PEP, we can read that $\langle\Delta \mathrm{PEP}\rangle_{\mathrm{A}}=-11.29$ and $\langle\Delta \mathrm{PEP}\rangle_{\mathrm{Sh}}=-5.51$; consequently, the corresponding normalized values are $\langle\Delta \mathrm{PE} P\rangle_{\mathrm{A}}=-1$ and $\langle\Delta \mathrm{PE} \mathrm{P}\rangle_{\mathrm{Sh}}=-5.51 /|-11.29|=-0.488$.

With these normalised values, the correlation factor $\mathrm{R}$ can be computed for all the couples of cardiovascular-emotional observables. For example, for the two couples (A,Sh) and

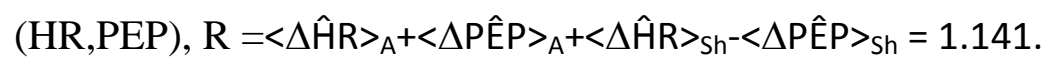

The 12 values of $\mathrm{R}$ for this experiment are reported in the table below:

\begin{tabular}{|l|l|l|}
\hline & A, Sh & Sh, A \\
\hline HR, PEP & 3.14 & 0.16 \\
\hline HR, CO & 0.28 & 3.03 \\
\hline PEP, CO & -0.86 & 1.88 \\
\hline CO, PEP & 0.86 & -0.62 \\
\hline HR, CO & 0.28 & 3.03 \\
\hline PEP, HR & 0.86 & 0.16 \\
\hline
\end{tabular}

Table 3. Values of $\mathrm{R}$ for all couples of cardiovascular/emotional observables computed from the data of (Kassam and Mendes 2013)

The last step consists in comparing the values of the $\mathrm{CHSH}$ correlation factor $\mathrm{R}$ obtained in this table with the Bell bound and the signaling bound $\beta$, as explained in section III.2. In this 
small part of the meta-analysis (12 trials on a total of 164), one can read that 9 values of $\mathrm{R}$ satisfy the Bell bound and then the signaling bound $(|R| \leq \beta)$ while 3 values violate the Bell bound (but could also violate the signaling bound $\beta$, which has not be computed here). As reported in section III.2, the global result (on 164 trials) shows the quasi-impossibility to interpret causally the studied psychophysical correlations with a high confidence level.

On behalf of all authors, the corresponding author states that there is no conflict of interest.

\section{References}

Aerts, D., Sozzo S., Gabora L. and Veloz T. 2011. Quantum structure in cognition: Fundamentals and Applications. ICQNM 2011: The Fifth International Conference on Quantum, Nano and Micro Technologies.

Aerts D, Sassolini de Bianchi M, Sozzo S and Veloz T. 2020. Foundations of science. Special Issue: International Symposium "Worlds of Entanglement", Vol 25 (1).

Alexander F. 1950. Psychosomatic medicine: Its principles and applications. New York: Norton.

Atmanspacher H. 2003. " Mind and matter as asymptotically disjoint, inequivalent representations with broken time-reversal symmetry. BioSystems 68, p. 19-30.

Atmanspacher H., Römer H., and Walach H. 2002. Weak quantum theory: Complementarity and entanglement in physics and beyond. Foundation of Physics 32, 379-406.

Baumgartner, M. 2010. Interventionism and Epiphenomenalism, Canadian Journal of Philosophy 40: 359-383.

Beck F. and Eccles J. 1992. Quantum aspects of brain activity and the role of consciousness. Proceedings of the National Academy of Sciences of the USA 89: 11357-11361.

Bell J.S. 1964. On the Einstein Podolsky Rosen paradox. Physics 1, 195.

Broom B. 2007. Meaning-full Disease. Ed. Karnak

Buhrman H. and Massar S. 2005. Causality and Tsirelson's bounds. Phys. Rev. A, 72.

Busemeyer J. R. and Bruza P. 2012. Quantum Models of Cognition and Decision. Cambridge University Press.

Canon W. B. 1927. The James-Lange theory of emotions: A critical examination and an alternative theory. American Journal of Psychology, 39, 106-124.

Chalmers D. 2007: The Hard Problem of Consciousness, in The Blackwell Companion to Consciousness. (Max Velmans et Susan Schneider: Blackwell Publising, 225-235.

Changeux, J-P. 1983. L'Homme neuronal. Paris: Fayard.

Clauser J. F., Horne M. A., Shimony A. and Holt R. A. 1969: Proposed Experiment to Test Local idden-Variable Theories, Phys. Rev. Lett. 23, 880. 
Crick F. 1994. Astonishing Hypothesis: The Scientific Search for the Soul. Scribner.

Damasio A. 1999. The feeling of what happens: Body et Emotions in the making of Consciousness. New York : Harcourt Brace. French trans. Le sentiment même de soi. Corps, émotions, conscience. Paris : Odile Jacob, 1999.

Darwin C. 1872. The Expression of the Emotions in Man and Animals. London: John Murray.

Davidson D. 1970: Mental Events, in Actions and Events, Oxford: Clarendon Press, 1980

Dehaene S. et Changeux J. P. 2004: Neural mechanisms for access to consciousness, in Michael Gazzaniga et al., The Cognitive Neuroscience III. Cambridge: MIT Press.

Dennett D. 1991. Consciousness Explained. Boston: Little, Brown.

Descartes R. 1641. Méditations métaphysiques, méditation IV, in Oeuvres complètes, t. III, éd. Ch. Adam et P. Tannery. Paris : Vrin, 1996.

Descartes R. 1644. Principes de Philosophie.

Edelman G. 2000. Biologie de la conscience. Odile Jacob. Traduction française de Bright Air, Brillant Fire: On the Matter of Mind. Basic Books 1992

Espagnat d' B. 1994. Le réel voilé. Paris : Fayard.

Geringer JM, Madsen KM and Gregory D. 2004. A fifteen-year history of the Continuous Response Digital Interface: Issues relating to validity and reliability, Bul. Council for Reserch in Music Education.

Groddeck G. 1923. Le livre du ça. Ré-édition, Paris : Gallimard 1976.

Heinroth J. 1818. Lehrbuch der Störungen des Seelenlebens oder der Seelenstörungen und ihrer Behandlung Leipzig: Vogel.

Jackson F. 1996. Mental Causation, Mind, 105: 377-413.

James J. W. 1884. What is an Emotion? Mind 9:188-205.

Janssen T. 2006. La solution intérieure. Paris: Fayard.

Kassam K.S. and Mendes W. B. 2013. The Effects of Measuring Emotion: Physiological Reactions to Emotional Situations Depend on wether Someone Is Asking. Plos One, June 05.

Kim J. 2005. Physicalism, or something near enough. Princeton University Press.

Kim J. 2006. Philosophy of Mind. Boulder: Westview Press.

Kistler, M. 1998. Reducing Causality to Transmission, Erkenntnis (48): 1-24.

Koch Ch. 2004. The Quest for Consciousness. Roberts \& Company Publishers. French translation by Christophe Pallier, ed. Odile Jacob 2006.

Lang P.J. 1979 A bio-informational theory of emotional imagery. Psychophysiology 16:495512.

Lange C. 1885. On Emotions: A Psycho-Physiological Study. French translation by Georges Dumas Les émotions: étude psychophysiologique, Paris:Félix Alcan, coll. «Bibliothèque de philosophie contemporaine», 1895.

Lazarus R. 1991. Emotion and adaptation. New York: Oxford University Press. 
Leopold D.A. and Logothetis N.K. 1996. Avtivity changes in early visual cortex reflects monkeys' percepts during binocular rivalry». Nature 379 : 549-553.

Levine J., Materialism and Qualia: The Explanatory Gap, in: Pacific Philosophical Quarterly, vol. 64, no. 4, October, 1983: 354-361.

Luo H.C., Jia Y.K. et al. 1985. Electroacupuncture vs Ametriptyline in the treatement of depressive states. Journal of Traditional Chinese Medicine 5: 3-8.

Mattuck, R. 1980. Une théorie quantique de l'interaction entre la conscience et la matière. In Science et Conscience, les deux lectures de l'univers, Editions Stock, Paris, pp. 77-96.

Nagel T. 1974: What Is It Like to Be a Bat? Philosophical Review, 83: 435-50.

Minsky M. 1988. La Société de l'Esprit. Paris: InterEditions.

Schachter S. and Singer J. 1962. Cognitive, social and physiological determinants of emotional state. Psychol. Rev. 69: 379-399.

Schrödinger E. 1935. Discussion of Probability Relations between Separated Systems, Proceedings of the Cambridge Philosophical Society, 31.

Searle J.R. 1997: The Mystery of Consciousness. The New York Review of Books.

Selye H. 1978. The stress of life. New York: Mc Graw-Hill.

Shapiro, L., and Sober, E. 2007. Epiphenomenalism | the Do's and the Don'ts, in P. Machamer and G. Wolters (eds.), Thinking about Causes, University of Pittsburgh Press, p. 235-264.

Sperry R. 1983. Science and Moral Priority, New York: Columbia University Press.

Spinoza, B. 1675 Ethics, English translation by Samuel Shirley, Hackett Publishing Company, Inc.2002.

Soon et al. 2008. Unconscious determinants of free decisions in the human brain, Nature Neuroscience 11, p.543 - 545.

Tononi G. 2007. The Information Integration Theory of Consciousness in The Blackwell Companion To Consciousness. Edited by Max Velmans and Susan Schneider. Oxford: Blackwell Publishing.

Trombini G. and Baldoni F. 2008. La psychosomatique. Coll. Psycho. Paris: Inpress.

Uzan, P. 2012. A quantum approach to the psychosomatic phenomenon: Co-ermergence and time entanglement of mind and matter. KronoScope, 12, 219-244.

Uzan P. 2013 Conscience et Physique Quantique. Vrin, collection Mathesis.

Uzan P. 2014a.On the Nature of Psychophysical Correlations, Mind and Matter (2):1.

Uzan P. 2014b. The Quantum-Like Approach of Psychosomatic Phenomena in Application. Axiomathes (24): 3.

Uzan P. 2016. Complementarity in Psychophysics. LNCS, Springer, Vol. 9535: 168-178.

Uzan P. 2017. Deciding the mind-body problem experimentally. Axiomathes, 27 (4):333-354.

Uzan P. 2020. The CHSH test can be used for all quantum-describable systems. Foundations of Physics, to be published. 
Kassam K.S. and Mendes W.B. 2013. The effects of measuring emotion: physiological reactions to emotional situations depend on whether someone is asking. Plos One.

Walach, H., \& Römer, H. 2011. Generalized entanglement - A nonreductive option for a phenomenologically dualist and ontologically monist view of consciousness. In H. Walach, S. Schmidt, \& W. B. Jonas (Eds.), Neuroscience, Consciousness and Spirituality (pp. 81-95).

Dordrecht: Springer.

Walach, H. and von Stillfried, N. 2011: Generalised Quantum Theory-Basic Idea and General Intuition: A Background Story and Overview. Axiomathes. 21, 185-209.

Walker, E. H. 1975. Foundations of Paraphysical and Parapsychological Phenomena, in L. Oteri (ed.) Quantum Physics and Parapsychology, New York: Parapsychology Foundation 1975.

Wegner, D.M. and Wheatley, T. 1999. Apparent mental causation: Sources of the experience of will. American Psychologist. 54 (7): 480-492.

Woodward, J. 2003. Making Things Happen, Oxford University Press. 